DOI: 10.1002/adma.((please add manuscript number))

\title{
Linear self-assembly of nanoparticles within liquid crystal defect arrays
}

By D. Coursault, J. Grand, B. Zappone, H. Ayeb, G. Lévi, N. Félidj and E. Lacaze*

[*] Prof. E. Lacaze Corresponding-Author, D. Coursault, Dr H. Ayeb

Institut des Nano-Sciences de Paris (INSP), UMR-CNRS 7588, Université Pierre et Marie

Curie-Paris 6, 4 pl Jussieu 75005 PARIS, France E-mail: emmanuelle.lacaze@insp.jussieu.fr

Dr. J. Grand, Dr. G. Lévi, Prof. N. Félidj.

Université Paris Diderot, Sorbonne Paris Cité, ITODYS, UMR CNRS 7086, 15 rue J-A de Baïf, 75205 Paris Cedex 13, France

Dr. B. Zappone

CNR-IPCF, Liquid Crystal Laboratory, Università della Calabria, cubo 33/B, Rende, 87036, Italy

Keywords: Liquid crystal; gold nanoparticles; self-assembly; Surface Plasmon Resonance; Optically Active Materials.

Nanostructured materials with tunable optical properties are of great interest in the perspective of optical transport of information. Gold nanoparticles (GNPs) exhibit unique optical properties due to Localized Surface Plasmon Resonance (LSPR), which are highly sensitive to the optical index of the embedding medium and the presence of neighboring GNPs. Linear (1D) assemblies of GNPs are particularly interesting due to their strong anisotropy. Various methods have been explored to create linear assemblies of nanoparticles but obtaining straight structures over a large scale remains challenging. A promising approach is the directed self-assembly of nano-objects within large-scale, ordered templates. ${ }^{[1,2]}$ This approach is particularly promising for liquid crystals (LC) which are able to rapidly selfarrange into large ordered anisotropic matrices which can contain linear structural defects. ${ }^{[3,4 \text {, }}$ ${ }^{5]}$ This kind of defect has been used so far as molt for polymerization. ${ }^{[6]}$ Concerning assembly of particles, many studies have been devoted to the trapping of $\mu \mathrm{m}$-sized particles by nematic and smectic defects. ${ }^{[7,8,9]}$ In nematic LC matrices, $\mu$-sized particles can create ${ }^{[10]}$ and interact with localized defects. ${ }^{[8,9,11]}$ In case of attraction between LC defects and particles, controlled large-scale arrays of particles can be obtained, leading to linear assemblies for the 
so-called nematic disclinations. ${ }^{[8,9,10]}$ However, these assemblies are not really straight and better trapping has been demonstrated for smectic defects compare to nematic ones, due to higher distortion energy associated with the former. ${ }^{[1]}$ Trapping of NPs by defects has been evidenced through the accumulation within defects, especially in LC blue phases. ${ }^{[12,13,14]}$ The following question thus arises: can the trapping of NPs be controlled at the nanoscale, for 1D geometry, even though NPs are expected to be too small to generate a long-range elastic LC distortion? The smallest particles around which LC distortions have been evidenced have a diameter of $125 \mathrm{~nm} \cdot{ }^{[15]}$ In this article we show that ordered linear arrays of defects in the smectic phase of a liquid crystal attract and organize NPs such as quantum dots (QDs) and GNPs. The well-defined alignments of single GNPs provide new means of tuning their optical response (e.g. their LSPR).

We have used films of smectic 8CB (4-n-octyl-4'-cyanobiphenyl) deposited on a solid substrate inducing planar anchoring of the LC director while a homeotropic (normal) anchoring was generated at the air interface. This produces a distorted texture, the so-called 'oily streaks', containing periodic curvature walls and disclination lines, perpendicular to the planar anchoring (Fig.1a). ${ }^{[4,5,15]}$ Due to the high energetic cost of their structural deformations, the defects core thickness is expected to be limited. ${ }^{[16]}$ We used well-defined defects orientations to induce anisotropic assemblies of NPs. We performed optical extinction, fluorescence microscopy, and scanning electron microscopy (SEM) to identify the role of defects and study the influence of the NPs concentration. Discrete dipole approximation (DDA) calculations were also performed for the analysis of extinction spectra. ${ }^{[17,19]}$ We have considered two substrates: (a) a freshly cleaved surface of crystalline molybdenum disulfide $\left(\mathrm{MoS}_{2}\right)$, semi conductive and opaque to light, and (b) transparent coatings of poly-vinyl alcohol (PVA) on glass, rubbed to induce planar anchoring. We used two types of NPs: (i) gold NPs (Aldrich) with a diameter of $5 \mathrm{~nm}$, coated with dodecanethiols to avoid aggregation 
in toluene solution, and (ii) fluorescent CdSe-ZnS QDs (Invitrogen) characterized by binary blinking fluorescence $\left(\begin{array}{llllll}\lambda & \operatorname{exc}=440 & \mathrm{~nm}, & \lambda & \mathrm{emi}=630 & \mathrm{~nm}) .\end{array}{ }^{[20]}\right.$
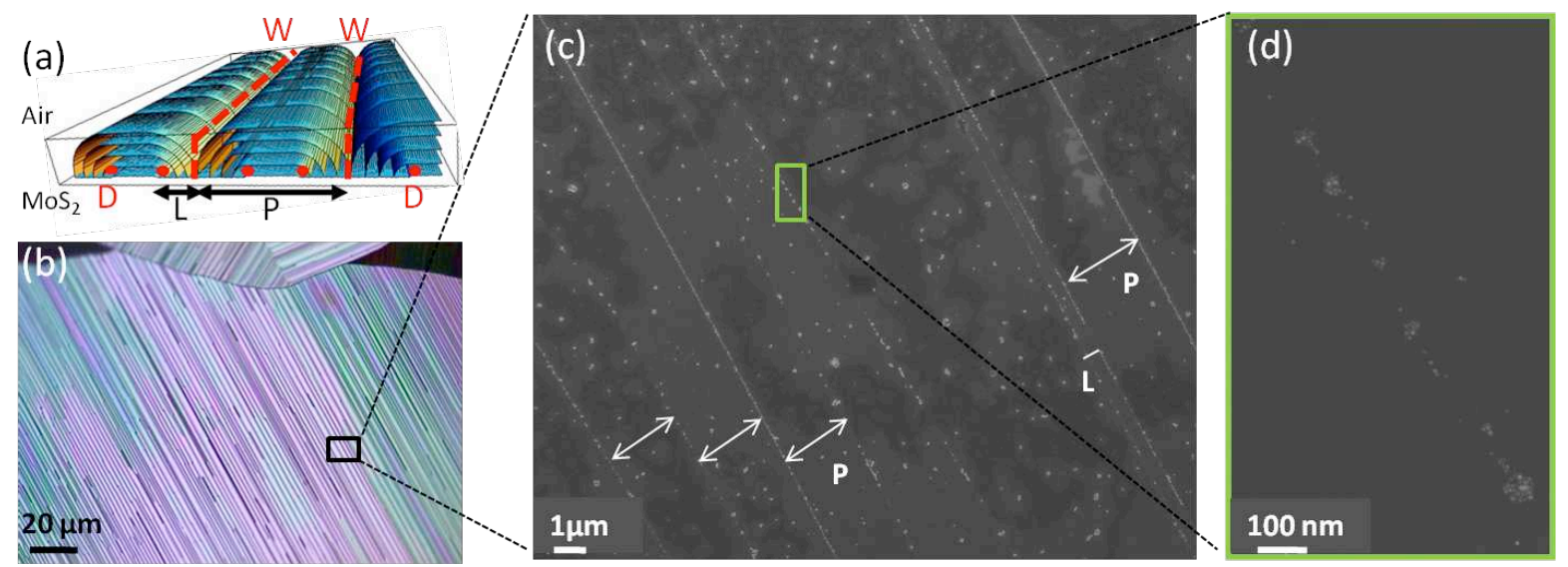

Figure 1.a. Schematic illustration of a linear array of straight parallel oily streaks in which only smectic layers are represented, with disclination lines (D) and curvature walls (W). b. Polarized optical microscopy image of a single array of a $300 \mathrm{~nm}$ thick film of $8 \mathrm{CB}$ on $\mathrm{MoS}_{2}$. c. SEM image of the $\mathrm{MoS}_{2}$ substrate with GNPs chains. d. Enlarged view of a chain.

In order to visualize the GNPs by SEM, we first started experiments on the $\mathrm{MoS}_{2}$ substrate. When GNPs were deposited without LC, SEM images revealed dense GNPs areas homogeneously dispersed on the substrate (not shown). They correspond to close-packed GNPs separated by $1.5 \mathrm{~nm}$ on average, due to alkylthiols chains interdigitation. A solution of $3 \times 10^{16} \mathrm{GNPs} / \mathrm{L}$ in toluene was then mixed with $5 \mathrm{mM}$ of $8 \mathrm{CB}$ and spin-coated 25 times to obtain a large number of GNPs and a LC film thickness of 300nm. ${ }^{[3]}$ The sample was heated 2 minutes at $60^{\circ} \mathrm{C}$ in a second step to allow appearance of $8 \mathrm{CB}$ oily streaks when cooling back to room temperature. Polarized optical microscopy images did not show textural changes in the $8 \mathrm{CB} / \mathrm{GNPs}$ array compared to pure $8 \mathrm{CB}$ (Fig. 1b). In a third step $8 \mathrm{CB}$ was eliminated by heating the substrate at $60^{\circ} \mathrm{C}$ for one week. The lamellar texture of $\mathrm{MoS}_{2}$ indeed allows formation of $8 \mathrm{CB}$ monolayers at each basal planes, ${ }^{[21]}$ leading to a draining of $8 \mathrm{CB}$ which systematically occurs within $\mathrm{MoS}_{2}$ in contrast to other substrates, like rubber polymer on glass. SEM images showed GNPs arranged in straight parallel chains with lengths up to several hundreds of $\mu \mathrm{m}$ (Fig. 1c). The typical distance between chains (from 1.5 to $2.2 \mu \mathrm{m}$ ) 
was of the same order than the distance between walls, P, (Fig. 1a), imposed by the LC film thickness. ${ }^{[3]}$ We occasionally observed double chains of GNPs separated by a smaller distance, L, (Fig. 1c), comparable to the typical walls-disclination distance (about $300 \mathrm{~nm}$ ). ${ }^{[15]}$ Disclinations can also trap GNPs, though less efficiently than walls. We observe other areas with randomly oriented thicker alignments of GNPs (Fig. 2c). As the planar anchoring on $\mathrm{MoS}_{2}$ is multi-directional, oily streaks are oriented in domains corresponding to different azimuthal (in-plane) anchoring directions (Fig. 2a), ${ }^{[21]}$ coexisting on the same cleavage plane of $\mathrm{MoS}_{2}$. We identify regions between randomly oriented alignments of GNPs with small anchoring domains of $\mathrm{MoS}_{2}$ and alignments of GNPs with domain (grain) boundaries. The density of GNPs is the largest in regions where several domains meet each other (arrow on Fig. 2c). Therefore, GNPs are preferentially trapped in grain boundary regions where the LC director is highly distorted to accommodate the disorientation between 3 azimuthal directions. The migration of GNPs from the oily streaks region to grain boundaries shows that different types of defects have a different 'trapping efficiency': Grain boundaries are more efficient than walls and disclinations to trap NPs, and disclinations turns out to be the least efficient "trapping system" of all.

Fig 1.d shows both clusters and single GNPS along a same line. This suggests that avoiding the aggregation of clusters leads to chains of individual GNPs in oily streaks. Isolated NPs are observed on Fig. 1, which means that the NPs concentration on the substrate must be adjusted. We have then decided to control the NPs concentration using drop-casting on a rubbed PVA polymer substrate. This kind of substrate presents the advantage of having a unique planar anchoring direction for $8 \mathrm{CB}$. The deposition of a drop of $8 \mathrm{CB}$, immediately followed by a 2 minutes heating at $60^{\circ} \mathrm{C}$ during solvent evaporation, makes it possible to avoid the formation of NPs clusters. These clusters may be formed using spincoating, most likely during the formation of the smectic film prior to the appearance of the 
oily streaks (Figure 1c). We have then performed a study of the influence of the nanoparticles concentration on rubbed PVA polymer substrate.
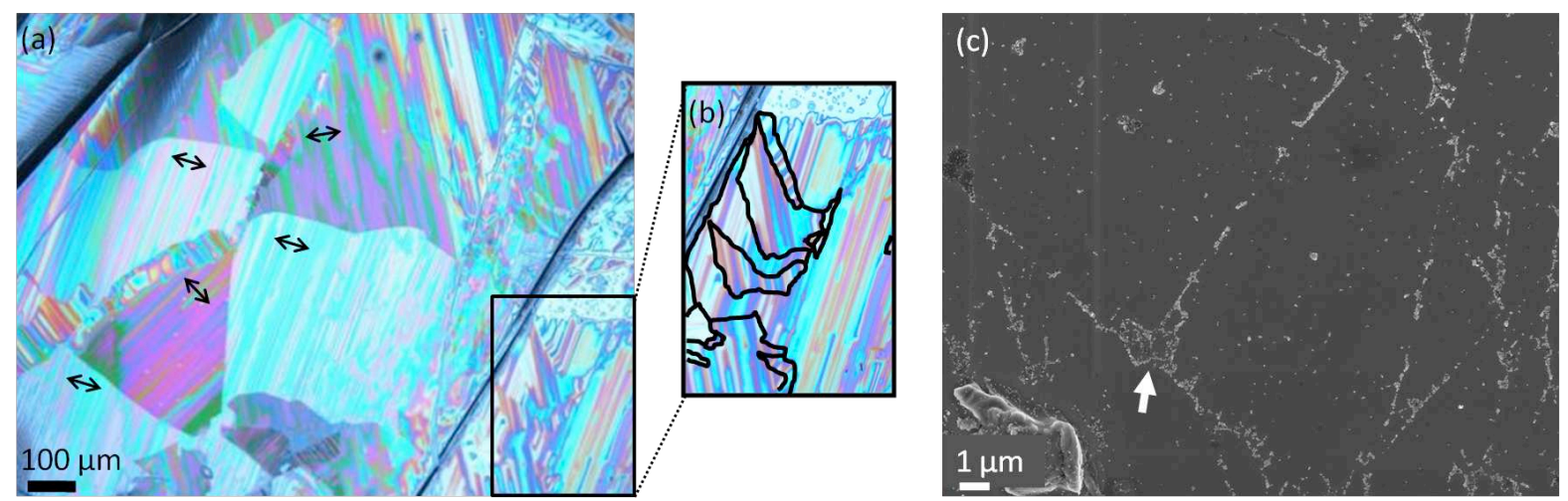

Figure 2. a. Polarized Optical Microscopy image of multiple anchoring domains of 8CB on $\mathrm{MoS}_{2}$ (the arrows show anchoring direction). b. Enlarged view with a high density of grain boundaries. c. SEM image of the $\mathrm{MoS}_{2}$ with thick alignments of GNPs aligned along the grain boundaries. The arrow points to a region where 3 grain boundaries meet each other.

At low concentration, we have considered QDs of the same diameter (5 nm) as GNPs which can be observed in-situ by fluorescence microscopy. We prepared a solution containing $3.5 \times 10^{12}$ QD.L $L^{-1}$ and $5 \mathrm{mM} 8 \mathrm{CB}$ in toluene. We deposited a $30 \mu \mathrm{l}$ drop on rubbed PVA polymer, to form a 300nm thick film with an average surface density of 0.7 QD. $\mu m^{-2}$. After cooling back from $60^{\circ} \mathrm{C}$ in the smectic phase, array of oily streaks appeared (Fig. 3a). Using fluorescence microscopy, without QD, no chain was visible. In presence of QDs, straight chains of blinking fluorescent spots parallel to the oily streaks (Fig. 3b) are visible. The distance between neighboring chains ranges between 0.64 and $7.5 \mu \mathrm{m}$, which is of the same order than the distances between walls (Fig. 3a, 1c). The wide range of distances is due to variations in the $8 \mathrm{CB}$ thickness. A careful analysis of the blinking QDs allows to identify one bright spot on Fig. 3b as being 2 QDs in average. This is consistent with average spot size which ranges from 0.5 to $1.7 \mu \mathrm{m}$, that is 1 to 3 times the calculated QD airy diameter $(549 \mathrm{~nm}$ for $\lambda_{\text {emi }}=630 \mathrm{~nm}$ ). From the density of spots in Fig. 3b, we calculate a surface density of 0.8 QD. $\mu \mathrm{m}^{-2}$, which is very close to the estimated average density of 0.7 QD. $\mu \mathrm{m}^{-2}$. Therefore, almost all QDs are trapped in the defects, showing that walls are very efficient at trapping and 
directing an assembly of NPs much smaller than the particles used in previous studies.

$[9,15]$

The length of the QDs chains is given by the one of the oily streaks, longer on PVA compare to $\mathrm{MoS}_{2}$ because they do not end in grain boundaries, leading to chains of several hundreds of micrometer, only interrupted by "dislocation-like" regions which accommodate thickness variations. $^{[5]}$

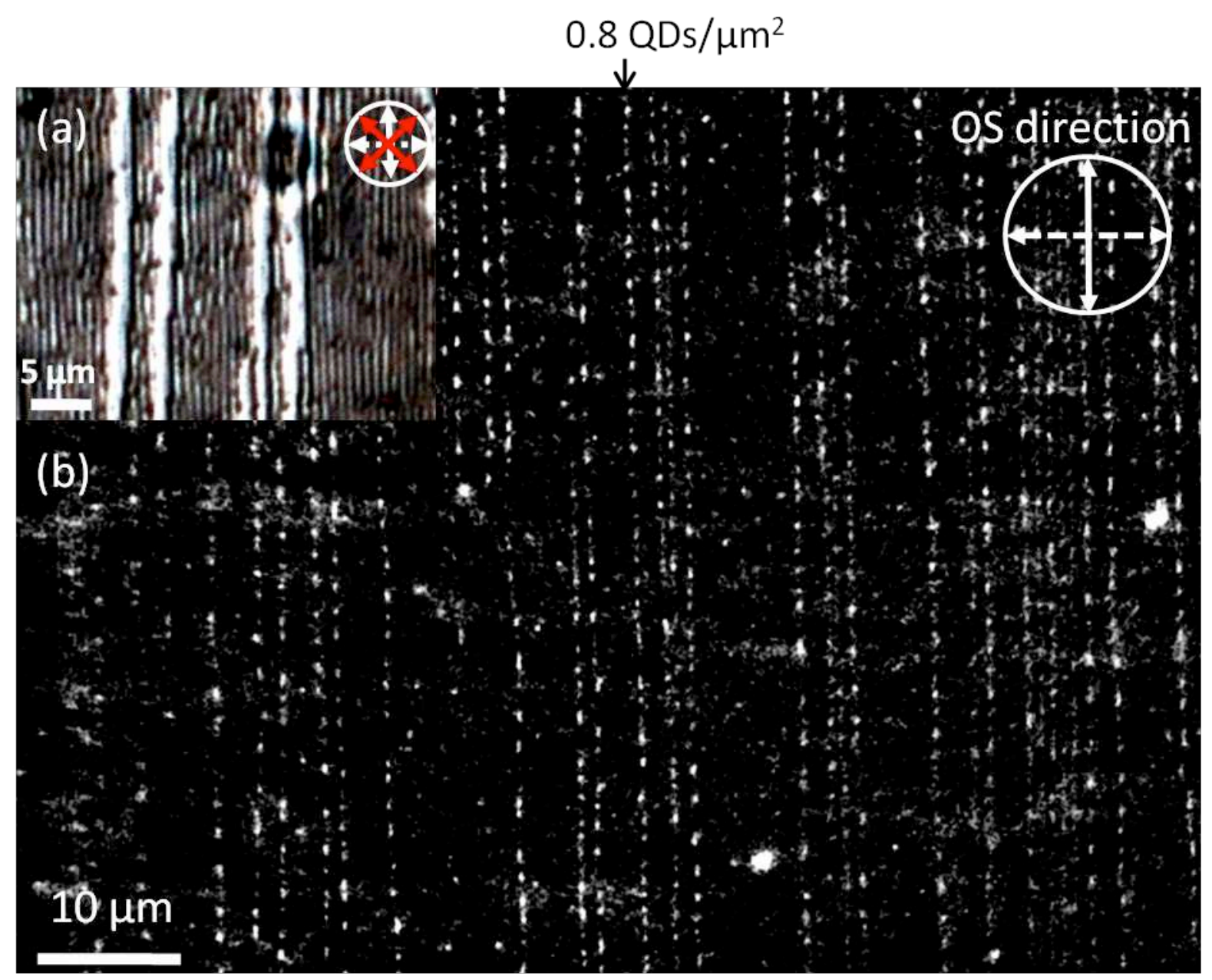

Figure 3. a. Polarized Optical Microscopy image of an array of oily streaks formed in a thin film of $8 \mathrm{CB} / \mathrm{QDs}$ on rubbed PVA. Red crosses show the directions of polarization, the dashed white arrow the anchoring direction and the solid white arrow the oily streaks direction b. Image of an array obtained by unpolarized fluorescence microscopy on the same film, with QDs.

To evidence inter-particle coupling and also the NPs alignment by a different mean, we replaced the QDs by GNPs at a concentration four orders of magnitude larger, $3 \times 10^{16}$ GNP.L ${ }^{-1}$ We then measured the localized surface plasmon resonance (LSPR) of the GNPs in 8CB. When incident light was polarized perpendicular to the oily streaks (Fig. 4a, black 
curve), the extinction peak was very similar to the one of GNPs dispersed in pure toluene ( $\lambda$ $=516 \mathrm{~nm}$, Fig. $4 \mathrm{a}$, dashed line). For parallel polarization, the LSPR is red-shifted by about 40 nm compared to the NPs in solution (Fig. 4a, red curve). It is well known that in isotropic media the LSPR moves to larger wavelengths as the refractive index increases. ${ }^{[20]}$ Due to the positive uniaxial birefringence of $8 \mathrm{CB}$, we expect to probe the ordinary index $\left(\mathrm{n}_{\mathrm{o}}=1.52\right)$ when the polarization is set parallel to the oily streaks and a refractive index varying between ordinary and extraordinary index $\left(\mathrm{n}_{\mathrm{e}}=1.67\right)$ for a polarization perpendicular to the oily streaks, depending on the localization of GNPs (Fig. 1a). Therefore, the 8CB birefringence could only lead to a blueshift of the LSPR for a polarization parallel to the oily streaks. The observed red shift is indicative of a different phenomenon, the coupling between GNPs preferentially along the oily streaks.
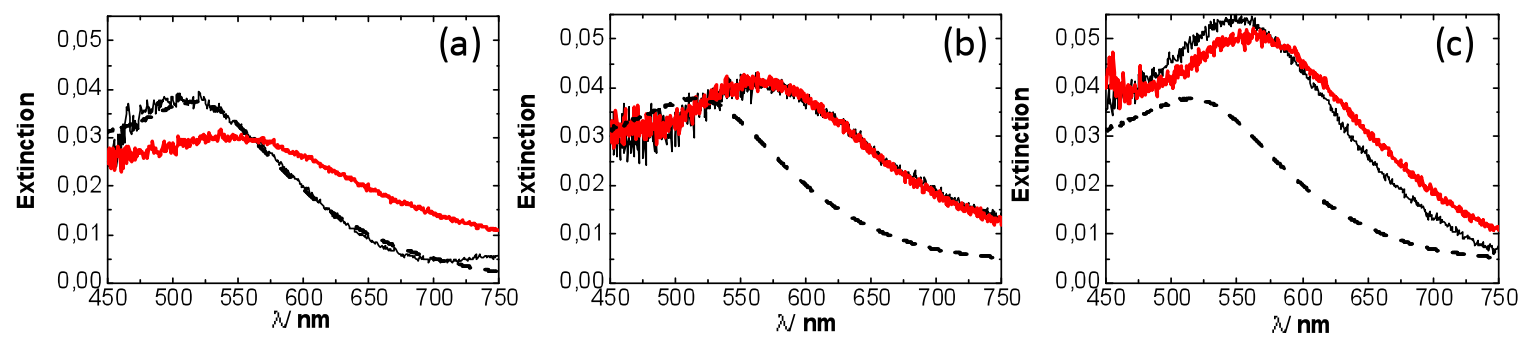

Figure 4. Extinction spectra recorded on rubbed PVA-polymer with an incident light polarization set either parallel (red curves) or perpendicular (black curves) to the oily streaks. The dashed curve was obtained for GNP/toluene solution ( $\left.3 \times 10^{16} \mathrm{GNP}^{-L^{-1}}\right)$, divided by 20

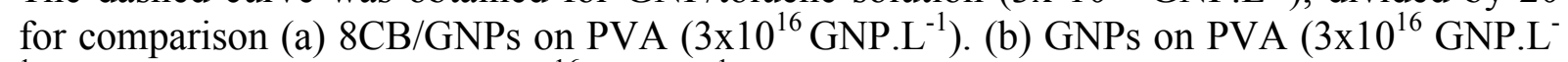
$\left.{ }^{1}\right)$. (c) $8 \mathrm{CB} / \mathrm{GNPs}$ on PVA $\left(6 \times 10^{16}\right.$ GNP.L $\left.\mathrm{L}^{-1}\right)$.

In contrast, no polarization anisotropy of the LSPR was observed for the same amount of GNPs deposited on PVA without LC (Fig. 4b). This is due to the presence of randomly oriented clusters of optically coupled GNPs, like on $\mathrm{MoS}_{2}$ without LC. ${ }^{[23]} \mathrm{We}$ have performed DDA calculations of the extinction spectra considering optically coupled gold/thiol core-shell GNPs. We have taken into account that: (a) the minimum distance between the metal cores of two GNPs was limited to $1.5 \mathrm{~nm}$ due to the presence of thiol coatings, (b) The extinction spectra measured for GNPs in pure toluene (dashed line) were blue-shifted by 16 
$\mathrm{nm}$ with respect to values calculated for uncoupled particles in toluene $\left(\mathrm{n}_{\text {toluene }}=1.497\right)$. The covalent bond at the gold-thiol interface may modify the dielectric constant of gold close to the interface. ${ }^{[19,20]}$ In the following we will only compare the experimental and theoretical variations of the extinction maxima with respect to the spectrum of GNPs in toluene. The calculated red-shift with respect to toluene was $10 \mathrm{~nm}$ for single GNPs embedded in $\mathrm{n}_{\mathrm{e}}$ and only $2 \mathrm{~nm}$ for $\mathrm{n}_{\mathrm{o}}$. The fact that, for perpendicular polarization (Fig. $4 \mathrm{a}$ ), no clear experimental shift is observed with respect to toluene, shows that GNPs feel an optical environment closer to $\mathrm{n}_{\mathrm{o}}$ than to $\mathrm{n}_{\mathrm{e}}$, as it should be in the vicinity of defects cores, associated with high local disorder for the LC director. This also shows that no coupling between GNPs occurs in the direction perpendicular to the oily streaks, which demonstrates a GNP organization in single linear chains parallel to oily streaks, without clusters like the ones on $\mathrm{MoS}_{2}$ (Fig. 1d). Moreover, for parallel polarization, no peak was observed around the wavelength for pure toluene $(516 \mathrm{~nm})$ showing that only a minority of GNPs is isolated on the substrate. A shift due to coupling between GNPs depends on the distance between particles and on the number of coupled particles. ${ }^{[24]}$ The larger width of the extinction peak for parallel polarization (Fig. 4a) shows that chains have a certain dispersion of length and/or separation between coupled GNPs. For five different areas on the same sample, the peak was red-shifted between $34 \mathrm{~nm}$ and $44 \mathrm{~nm}$. Calculated extinction spectra for GNP chains, in $n_{o}$ with a number of particles separated by $1.5 \mathrm{~nm}$ of thiol, going from 2 to infinity gave red shifts ranging from $17 \mathrm{~nm}$ to 40 $\mathrm{nm}$ with respect to the toluene reference. Long chains of closed-packed GNPs, longer than 8 GNPs, may consequently be formed in majority. The shift value is only limited by the minimal separation between GNPs, associated with direct contact between thiol coatings. These results finally demonstrate that well-oriented $1 \mathrm{D}$ chains are formed, in agreement with results obtained for fluorescent QDs, which must be localized in regions as small as $5 \mathrm{~nm}$, within oily streaks defects. Doubling the GNPs concentration $\left(6 \times 10^{16}\right.$ GNP.L $\left.^{-1}\right)$ leads to a red-shift for both polarizations: $36 \mathrm{~nm}$ for perpendicular one and $50 \mathrm{~nm}$ for parallel one (Fig. 
4c). The smaller red-shift observed for perpendicular polarization shows that a limited assembly of coupled GNPs occurs also perpendicular to oily streaks, corresponding to an overall shape still elongated along the oily streaks direction similar to a rod or a ribbon. Therefore, the anisotropy remains effective at high concentration, when the most stable traps within defects core have already been occupied. Variations of concentration allow tuning the extinction shift between perpendicular polarizations, in relation with the length and width of ribbons. A maximum value of $44 \mathrm{~nm}$ has been measured so far for single chains.

Our result agrees with the idea that NPs behave as impurities: they are stabilized by the gain in defects core energy in defects like wall, whereas the trapping of particles larger than the defect core is also due to a gain in elastic energy. ${ }^{[12,13,25,26]}$ The core energy per unit area of the wall is of the order of $\mathrm{K} / \mathrm{l}$, where $\mathrm{K}=10^{-11} \mathrm{~J} \cdot \mathrm{m}^{-1}$ is the curvature modulus, $\mathrm{l}=1 \mathrm{~nm}$ is the penetration length, and the core size is of the order of $1 .^{[11,17]}$ The trapping potential is $\mathrm{a}^{2} \mathrm{~K} / 1 \approx 80 \mathrm{kT}$, where $\mathrm{a}=5 \mathrm{~nm}$ is the NP diameter, leading to a very stable and localized trapping. The small size of the defect is specific to smectic defects, as opposed to nematic defects, and constitutes a key point for successful aligning trapping of NPs. A limit size of the NPs is expected below which gain in core energy becomes smaller than thermal energy.

Possibly, the trapping sites correspond to edge dislocations within walls joining highly disoriented layers, ${ }^{[5]}$ which are expected at the base of the oily streaks. ${ }^{[16]}$ Dislocations have also been observed within disclinations on $\mathrm{MoS}_{2}$ surfaces. ${ }^{[16]}$ As the concentration increases, the chains are transformed in aligned ribbons suggesting a profile of trapping efficiency, associated with a gain in elastic energy outside the defects cores, in the direction perpendicular to the defects, which becomes progressively occupied by NPs.

The causes of NP trapping in defects are complex and yet to be clarified. On one hand, the formation of chains may be the result of a random process of diffusion and aggregation of NPs. In nematics containing nm-size macromolecules, this process leads to the segregation of molecules into defects, but also leaves a significant fraction of molecules outside the defects 
in the form of aggregates. ${ }^{[26]}$ In contrast, regions as wide as several $\mu \mathrm{m}$ between the defects appeared almost completely depleted of NPs on rubbed polymer substrate, as if NPs were attracted to the defects by a long-range interaction. In nematics, $\mu \mathrm{m}$-size particles are known to create defects and elastic distortions of the molecular director that produce forces between particles and defects. ${ }^{[8,9]}$ It is known that NPs with alkylthiols (inducing homeotropic anchoring) produce a disordering effect in LCs. ${ }^{[27]}$ In the smectic phase, the distortion around NPs may be enhanced. ${ }^{[28]}$ The question remains whether NPs produce a local disturbance of the smectic film, on a scale comparable with their size, or they create a distortion over a much larger scale that may be coupled with the long-range elastic distortion associated with defects. In the latter case, the distortion may produce a 'guided' diffusion of the NPs towards the singularity of the distorted texture, in analogy with $\mu \mathrm{m}$ size particles in the nematic phase.

Three kinds of defects have been identified on $\mathrm{MoS}_{2}$, all of which constitute stable trapping sites for NPs: grain boundary, curvature wall, and disclination, in order of decreasing trapping efficiency. This is compatible with an attraction between GNPs and defects related to the range of the distortion within the LC bulk. Grain boundaries involve the largest disorientation of the layers at opposite side of the boundary. Straight vertical walls (Fig. 1(a)) are more effective than disclination, because they have an extension equal to the LC film thickness whereas the disclinations on $\mathrm{MoS}_{2}$ have a diameter of the order of $100 \mathrm{~nm} .{ }^{[16]}$

In conclusion, we have demonstrated that smectic defects can constitute efficient traps for NPs as small as 5nm, due to the combination of highly localized trapping sites characterized by deep trapping energy profile and large-scale elastic deformations induced in the LC film. In oily streaks, they can be precisely oriented along directions of anisotropy imposed by the substrate. This allowed us to produce arrays of straight chains of single NPs separated by distances varying between some $\mu \mathrm{m}$ and $1.5 \mathrm{~nm}$ when the NP concentration is increased by four orders of magnitude, finally transforming into thick chains when it is further 
increased. As a consequence, LSPR of GNPs/LC systems can be tuned by polarization, in a matter controllable by the concentration of GNPs. These results also open the route for alignments of a vast number of NP types.

\section{Experimental}

The optical properties of GNPs were investigated with a LOT Oriel MS260i spectrometer coupled to an upright optical microscope (Olympus BX 51) to probe a $40 \mathrm{x} 40 \mu \mathrm{m}^{2}$ area. QDs fluorescence was investigated with an inverted Olympus IX71 optical microscope with an oilimmersed objective $(\mathrm{x} 100, \mathrm{NA}=1.4)$ connected to a CCD camera. GNPs visualization was performed on a field emission SEM, Zeiss Supra 40.

\section{Acknowledgements}

We thank Agence Nationale de la recherche (ANR) for financial support corresponding to projects ANR-07-Nano-032 and ANR-09-Nano 003 and Laurent Coolen for fluorescence microscopy experiments and discussions.

Received: ((will be filled in by the editorial staff))

Revised: ((will be filled in by the editorial staff)) Published online: ((will be filled in by the editorial staff)) [1] M. Grzelczak, J. Vermant, E.M. Furst, and L.M. Liz-Marzan, ACS Nano, 2010,4, 3591.

[2] G. M. Whitesides, J. K. Kriebel, and B. T. Mayers et al., in Nanoscale Assembly, (ed:D.J. Lockwood), Chem. and Mat. Sci. Nanostruct. Sci. and Tech., Springer, 2005, ch. 9.

[3] J.P. Michel, E. Lacaze, M. Alba, M. de Boissieu, M. Gailhanou, and M. Goldmann, Phys. Rev. E, 2004,70, 11709.

[4] B. Zappone and E. Lacaze Phys. Rev. E, 2008,78, 061704.

[5] B. Zappone, E. Lacaze, H. Ayeb, M. Goldmann, N. Boudet, P. Barois, and M. Alba, Soft Matter, 2011, 7, 1161. 
[6] Y.H Kim, D.K.Yoon, H.S.Jeong, O.D. Lavrentovich and H.T. Jung, Adv. Funct. Mat., 2011,21, 610 .

_[7] D. K. Yoon, M. C. Choi, Y.H. Kim, M. W. Kim, O. D. Lavrentovich and H-T Jung, Nat. Mat., 2007,6, 866.

[8] J. B. Fleury,D. Pires, and Y. Galerne, Phys. Rev. Lett., 2009, 103, 267801.

_[9] D. Pires, J-B. Fleury, and Y. Galerne, Phys. Rev. Lett., 2007, 98, 247801.

[10] P. Poulin, H. Stark, T. C. Lubensky and D. A. Weitz, Science,1997, 275, 1770.

[111] A. Jakli, B. Senyuk, G. Liao, O.D. Lavrentovich, Soft Matter, 2008, 4, 2471

[112] H.Yoshida, Y. Tanaka, K. Kawamoto, H. Kubo, T. Tsuda,

A. Fujii, S. Kuwabata, H. Kikuchi, and M. Ozaki, Appl. Phys. Express, 2009, 2, 121501.

[13] E.Karatairi, B. Rozic, Z. Kutnjak, V. Tzitzios, G. Nounesis, G. Cordoyiannis, J. Thoen,

C. Glorieux, and S. Kralj, Phys. Rev. E, 2010, 81, 041703.

_[14] H. K. Bisoyi and S. Kumar, Chem. Soc. Rev., 2011, 40, 306.

[15] M. Skarabott and I. Musevic, Soft Matter, 2010, 6, 5476.

[16] J.P. Michel, E. Lacaze, M. Goldmann, M. Gailhanou, M. de Boissieu, and M. Alba, Phys. Rev. Lett., 2006, 96, 027803.

[17] M. Kléman, Lines and Walls in Liquid Crystals, Magnetic Systems and Various Ordered Media, John Wiley \& Sons, 1983.

[18] G.A. Rance, D. H. Marsh, A. N. Khlobystov, Chem. Phys. Lett., 2008, 460, 230.

_[19] M. Nirmal, B. O. Dabbousi, M. G. Bawendi, J. J. Macklin, J. K. Trautman, T. D. Harris and L. E. Brus, Nature, 1996, 383, 802.

_[20] P. Mulvaney, Langmuir, 1996,12, 788.

[21] P. B. Johnson and R. W. Christy, Phys. Rev. B, 1972,6, 4370.

_[22] E. Lacaze,J.P. Michel, M. Goldmann, M. Gailhanou, M. de Boissieu, and M. Alba et al. Phys. Rev. E, 2004, 69, 41705. 
[24] H. Yockell-Lelievre, D. Gingras, R. Vallee, A. M. Ritcey, J. Phys. Chem. C, 2009, 113, 21293.

[25] N. G. Khlebtsov and L. A. Dykman, J. Quant. Spectrosc. Radiat. Transfer, 2010, 111, 1.

_[25] M. Ravnik, G.P.Alexander, J.M.Yeomans, S. Žumer, Proc. Natl. Acad. Sci. U. S. A., 2011, 108, 5188 .

_[26] D. Voloschenko, O.P. Pishnyak, S.V. Shiyanovskii, , O.D. Lavrentovich, Phys. Rev. E, 2002, 65, 060701

_[27] B. Rozic, V. Tzitzios, E. Karatairi, U. Tkalec, G. Nounesis, Z. Kutnjak, G.

Cordoyiannis, R. Rosso,E.G. Virga, I. Musevic, and S. Kralj, Eur. Phys. J. E, 2011, 34, 17.

_[28] R. Prathiba, K. Park, I. I. Smalyukh, and W. Park, Optics express, 2007, 17, 19459. 


\section{Table of contents}

Keyword: Liquid crystal; nanoparticles; self-assembly; Surface Plasmon Resonance; Optically Active Materials.

D. Coursault, J. Grand, B. Zappone, H. Ayeb, G. Lévi, N. Félidj and E. Lacaze*

\section{Title}

Linear self-assembly of nanoparticles within liquid crystal defect arrays.

ToC figure ((55 $\mathrm{mm}$ broad, $50 \mathrm{~mm}$ high, or $110 \mathrm{~mm}$ broad, $20 \mathrm{~mm}$ high $))$

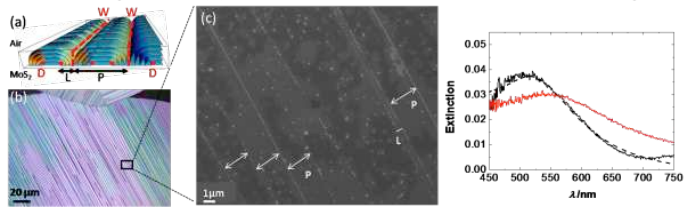

In presence of oriented smectic liquid crystal defects, hybrid systems nano-particles/liquid crystals form straight chains of nano-particles of length larger than tens of $\mu \mathrm{m}$ and width equal to one single nano-particle. The interparticle distance in a chain can be varied between few $\mu \mathrm{m}$ and $1.5 \mathrm{~nm}$, highlighting the control of optical absorption by light polarization monitored by gold nano-particles concentration. 